\title{
Two modifications of GNSS receiver system in simulation with Matlab
}

\author{
Sichao Li, Yuepeng Yan, Jinhai Li \\ Dept. of Electronics System Tech. \\ Institute of Microelectronics of CAS \\ Beijing, China \\ lisichao@ime.ac.cn; yanyuepeng@ime.ac.cn \\ lijinhai@ime.ac.cn
}

\begin{abstract}
Simulation with Matlab is a basic method in design of GNSS(global navigation satellite system) receiver. Based on the theoretic analysis of spreading code generator and carrier generator in GNSS, this paper proposed modified generators of both code and carrier in Matlab after considering the characteristic of Matlab. Under the condition of different cycles of spreading code and carrier, the modified generating methods had been tested. The results show that, compared with traditional ones, the speed of modified spreading code generator raises by more than 50 times, and the speed of modified carrier generator raises by more than 110 times.
\end{abstract}

Keywords-receiver of GNSS; simulation with Matlab; running time; spreading code generator; carrier generator

\section{INTRODUCTION}

GNSS(global navigation satellite system) has been applied widely in both military filed and civil market. The receivers are playing an important role in weapon guidance, vehicle navigation, mapping and so on[1,2]. Matlab provides libraries with profuse functions and convenient development environment, so simulating and testing in Matlab is a basic method to design the GNSS receiver. The GNSS signal consists of PRN(pseudo random noise) code, carrier and navigation data. The navigation data can be gained by BPSK demodulation, while the PRN code and carrier must be precisely synchronized with the satellite signal through local code and carrier generators[3]. When we simulate the GNSS in Matlab, these two kinds of generator will influence the efficiency of system and the rate of R\&D significantly. The traditional generators are based on DDFS(direct digital frequency synthesis)[3-7], the principle of which is that store the wave data at first, then according to the FCW(frequency control words) and output of accumulator, read wave data from memorizer. This method fits high speed integrate circuit[8]. The instruction of Matlab program is executed while being explained[9]. The sampling frequency of GNSS receiver is usually higher than $16 \mathrm{MHz}[5,10]$. As a result, it will take a great deal of time to run the program if DDFS is adopted and the sampling points are generated one by one. This paper analyzes the principle of both PRN code generator and carrier generator, and modifies the two generators under development environment of Matlab. Based on the novel schemes, the simulation system runs much faster.

\section{PRINCIPLE OF SIGNAL GENERATORS}

The traditional method of both generators has similar operation mode, which is on the basis of DDFS, and adopts NCO(numerically controlled oscillator) to control output. Fig. 1 shows the system structure. The software of system provides FCW $\mathrm{M}$ resulted from required frequency, then the accumulator calculates current digital phase and sends it to holding register. In the end, the data mapper selects the output value according to predetermined rule.

FCW M could be got from the following equation[3]:

$$
M=\frac{2^{N} f_{0}}{f_{s}}
$$

Where $\mathrm{N}$ is the length of holding register, $f_{0}$ is required frequency, $f_{s}$ stands for sampling frequency, and frequency resolution $\Delta f=\frac{f_{s}}{2^{N}}$.

To improve the code resolution, every chip can be divided into two half chips, i.e. two code phase. Each cycle of GPS C/A code consists of 1023 chips, so it has 2046 code phase. Thereby, the mapper of code generator need a phase counter, which is applied to record code phase. After that the FCW M

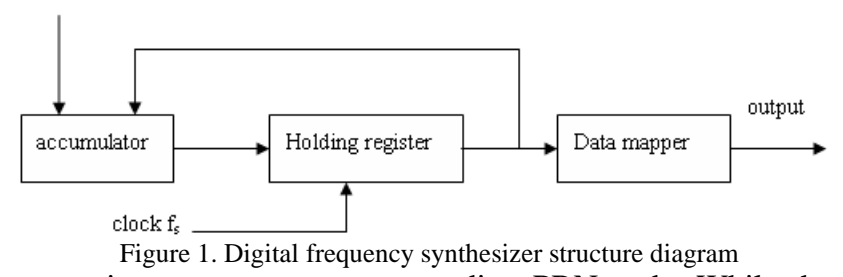
memorizer can output corresponding PRN code. While the digital phase of holding register overflows, the code phase counter will update.

For the carrier generator, if digital carrier employs $\mathrm{K}$ bits to quantize each point, the K MSB bits of digital phase will be suitable for data mapping. Different from the code generator, the carrier generator outputs two kinds of signal which are usually called I and Q. Table 1 illustrates the mapping scheme when $\mathrm{K}=3$. Evidently, while the digital phase of carrier generator overflows, the carrier would meet the next cycle. 


\section{THE MODIFIED SCHEME OF SIGNAL GENERATOR BASED ON MATLAB}

As shown in section II, when the traditional method is applied to generator PRN code and digital carrier in Matlab, the simulation program must adopt loop sentence and output the sampling point one by one. However, Matlab program is explained as it runs. The loop sentence may make certain instructions be explained a lot of times, so the GNSS program will run for a long time while there's lots of data. It's obviously that using vector instead of loop would let the system explain each instruction only once, and the operation time can be reduced very much.

\section{A. Modified algorithm of code generator}

According to the analysis of section II, while digital phase of code generator overflows, the PRN code will find the next phase, or else it will stay in current phase. Thus the output of code generator will follow the order of code phase strictly. Every sampling point corresponds with a digital phase, which indicates the point's location in corresponding code phase. If the simulation system records all the digital phase of current code cycle as well as the FCW of both current code cycle and next one, the digital phase of next code cycle will be calculated easily. On the basis of that, adjust the output of current cycle, and the output of next cycle can be received. In this way, the Matlab program replaces the loop with vector.

For the current code cycle, let $M_{1}$ stands for FCW, $\boldsymbol{S}_{\boldsymbol{1}}$ stands for output sequence, $\boldsymbol{P}_{1}$ stands for corresponding digital phase sequence, $T_{1}$ stands for the length of $\boldsymbol{P}_{\mathbf{1}}$. For the next code cycle, let $M_{2}$ stands for FCW, $\boldsymbol{S}_{2}$ stands for output sequence, $\boldsymbol{P}_{2}$ stands for corresponding digital phase sequence, $T_{2}$ stands for the length of $\boldsymbol{P}_{\mathbf{2}}$. The maximum of holding register is $C=2^{\mathrm{N}}-1$. If $M^{\prime}=M_{2}-M_{1} \geqslant 0$, the novel method could be realized as following:

1. Calculate the first element of $\boldsymbol{P}_{2}, \boldsymbol{P}_{2}(1)=\bmod \left(\boldsymbol{P}_{1}\left(T_{1}\right)+\right.$

TABLE I MAPS FOR $K=3$

\begin{tabular}{cccc}
\hline K MSB bits & Scope of quantization & $\mathrm{I}(\cos )$ & $\mathrm{Q}(\sin )$ \\
\hline 000 & $0^{\circ}-45^{\circ}$ & 2 & 1 \\
001 & $45^{\circ}-90^{\circ}$ & 1 & 2 \\
010 & $90^{\circ}-135^{\circ}$ & -1 & 2 \\
011 & $135^{\circ}-180^{\circ}$ & -2 & 1 \\
100 & $180^{\circ}-225^{\circ}$ & -2 & -1 \\
101 & $225^{\circ}-270^{\circ}$ & -1 & -2 \\
110 & $270^{\circ}-315^{\circ}$ & 1 & -2 \\
111 & $315^{\circ}-360^{\circ}$ & 2 & -1 \\
\hline
\end{tabular}

$\left.M_{2}, \quad C+1\right)$;

2. Calculate $\boldsymbol{P}_{2}=\boldsymbol{P}_{1}+\left(\boldsymbol{P}_{2}(1)-\boldsymbol{P}_{1}(1)\right)+M^{\prime} *\left[0: T_{1}-1\right]$, and record the phase larger than C. Let $\boldsymbol{S}_{2}=\boldsymbol{S}_{1}, T_{2}=T_{1}$;

3. If $\boldsymbol{P}_{2}\left(T_{2}\right)>C$, search forward from $\boldsymbol{P}_{2}\left(T_{2}\right)$ until the first digital phase $p 0$ is found which isn' t bigger than $C$, then reject every digital phase behind $p 0$ as well as the corresponding sampling points in $\boldsymbol{S}_{2}$; if $\boldsymbol{P}_{2}\left(T_{2}\right)<C$, calculate $p_{L}=\boldsymbol{P}_{2}\left(T_{2}\right)+L \cdot M_{2}, L=1,2, \ldots$, find the maximum $L_{\max }$ which lead to $p_{L} \leqslant C$. Combine $\left[p_{1} p_{2} \cdots\right.$ $\left.p_{L \max }\right]$ with $\boldsymbol{P}_{2}$, and complement $\boldsymbol{S}_{2}$ with $L_{\max } \boldsymbol{S}_{1}\left(T_{1}\right)$ s; if
$\boldsymbol{P}_{2}\left(T_{2}\right)=C$, skip this step;

4. For the other elements of $\boldsymbol{P}_{2}$, if they aren't bigger than $C$, keep them and the related output in $\boldsymbol{S}_{2}$ unchanged; otherwise, calculate the remainder of them based on $C+1$, with which they would be replaced, and change the corresponding output in $\boldsymbol{S}_{2}$ into the value of next code phase;

In accordance with above steps, the program could acquire $\boldsymbol{S}_{2}$ and $\boldsymbol{P}_{2}$. When $\boldsymbol{M}^{\prime}<0$, the program may generate PRN code in a similar way, and the difference is that the digital phase from step 2 becomes smaller, even negative. Each step can be executed by vector in Matlab, as a result, the instruction to be explained decrease significantly.

\section{B. Modified algorithm of carrier generator}

In the environment of Matlab, if the required frequency and sampling time sequence are known, the carrier sequence can be generated from $\cos (\mathrm{x})$ and $\sin (\mathrm{x})$ function, i.e. no carrier generator is needed. The output of both functions is floating point number which isn't quantized. In order to agree with the output of the generator, the program must translate them into fixed point number based on certain mapping rule.

Let $\boldsymbol{P}$ _carr represents carrier phase sequence. While 3 bits per point are employed in quantization, the carrier generator's modified scheme will be realized as following:

1. Calculate output sequences of both $\mathrm{I}$ and $\mathrm{Q}$ I_carr $=\cos \left(\boldsymbol{P} \_\right.$carr $), \boldsymbol{Q} \_$carr $=\sin \left(\boldsymbol{P} \_\right.$carr $)$;

2. Judge the sign of every element sign_I=sign(I_carr $)$, $\operatorname{sign} \_Q=\operatorname{sign}\left(Q \_\right.$carr $)$;

3. Judge whether or not the absolute value of each element in I_carr and Q_carr is bigger than $\frac{\sqrt{2}}{2}$, which is sine

value of $45^{\circ}$ angle. The results should be stored in vectors deci_I and deci_Q. Obviously, the elements of both deci_I and deci_Q are equal to 0 or 1 ;

4. Calculate carrier amplitude sequences after quantization I_out=deci_I+1, Q_out=deci_Q+1, and multiply them with the sign sequences of step 2 . The product sequence will be same with the output of carrier generator.

The new method gives up traditional generator, and every step can be finished by vector in Matlab. So it shortens the running time of system evidently.

\section{SIMULATION RESULTS}

According to the steps shown in section III, we simulate the novel schemes of both generators in Matlab. The traditional method has also been tested to compare with others. The simulation environment is as follows: HP G42 notebook PC, 2.67GHz double CPUs, 2G RAM, Matlab v7.9.0. PRN code is from 2\# satellite of GPS, whose nominal frequency is $1.203 \mathrm{MHz}$. IF of carrier is $4.092 \mathrm{MHz}$, and sampling frequency is $16367667 \mathrm{~Hz}$. The built-in functions of Matlab, tic\&toc, have been called to record running time.

The Doppler frequency of PRN code is usually about \pm $3 \mathrm{~Hz}[11]$, so we let it be even between $1 \mathrm{~Hz} 2 \mathrm{~Hz}$ when the 
code sequence updates. During every time of simulation three kinds of code, which are called Early, Prompt and Late separately, are generated at the same time. The results are shown in Fig.2. Compared with traditional scheme, the operation time of modified scheme decreases by one order of magnitude, and the rate of running rises by more than 50 times. What's more, the more code cycles are generated, the better performance could be gained.

The carrier's Doppler frequency isn't over $\pm 5000 \mathrm{~Hz}[11]$, and $2600 \mathrm{~Hz}$ is adopted in simulation. Based on the mapping rule in Table.1, I\&Q carriers are generated. The running time has been indicated in Fig.3, from which it can be seen that the modified method reduces the operation time by two orders of magnitude compared with traditional one, while the rate increases by more than 110 times. Similar to code scheme, the more cycles are generated, the faster program runs.

Both Fig.2 and Fig.3 show that, the modified schemes could improve the efficiency of simulation system and shorten operation time markedly, which proves the superiority of new algorithm and the validity of realization process.

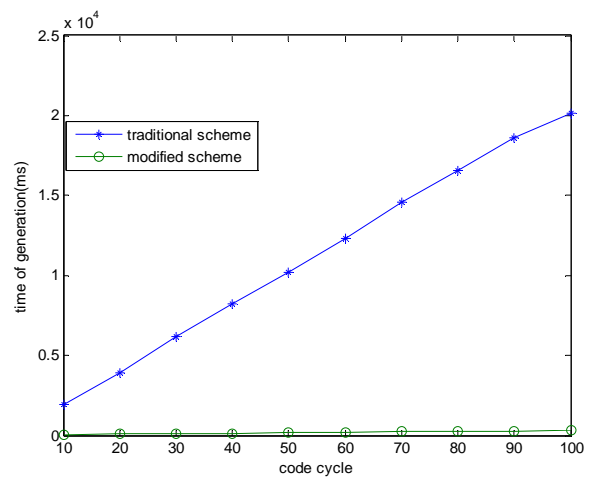

Figure 2. Operation time of code generator of two schemes.

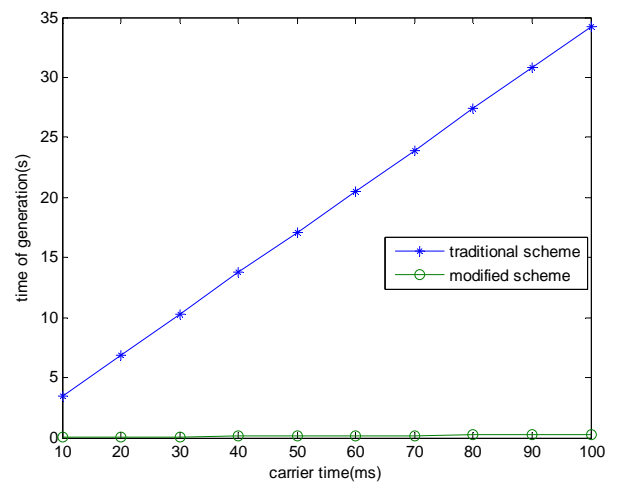

Figure 3. Operation time of carrier generator of two schemes

\section{CONCLUSIONS}

Adopting Matlab to simulate the system has taken a good importance in designing GNSS receiver. Through the theoretical analysis towards to code and carrier generators, considered on characteristics of Matlab, this paper proposes modified schemes of both signal generators. By comparison with the traditional method, the novel methods accelerate the operation of program and raise the efficiency of simulation.

\section{ACKNOWLEDGMENT}

This study was funded by the NHTRDPC-863 Project (No. 2001AA040102).

\section{REFERENCES}

[1] Shen Qiang, He Xin. GPS Positioning-Based Trajectory Parameter Estimation and Ejection Point Self-Adapting Control Method[C]// KAM'09.2009:194-197.

[2] Abdemalik B, Abderrahim B. Towards supporting GPS-unequipped vehicles in inter-vehicle geocast[C] //LCN '03. 2003: 766-767

[3] Elliott D K, Christopher J H. Understanding GPS Principles and Applications[M]. Boston: Artech House, 2006

[4] WU Lijing. Research on NCO and acquisition algorithm of GPS receiver in high dynamic[D]. Beijing: Beijing University of Posts and Telecommunications, 2009

[5] LI Sichao. Research on key technology of baseband signal processing of HDGPS receiver[D]. Beijing: Institute of Microelectronics of the Chinese Academy of Sciences, 2010

[6] ZOU Erning, XIE Zhongping. Design and implementation of the low frequency signal generator based on the method of direct digital frequency synthesis[J]. Process Automation Instrumentation, 2011, 32(3): $61 \sim 67$

[7] WANG Lei, LIU Zhenxing. Design of frequency synthesizer based on DDS and PLL.Information \& Communications(J), 2009, (3):11 16

[8] WANG Weiquan, LIU Changxiao. Implementation of C/A code generator in GPS with FPGA[J]. Journal of Guilin University of Electronic Technology(J),2006,26(5): 347 350

[9] DONG Jun.Characteristics and application of Matlab language[J].Journal of Jilin Province Economic Management Cadre College, 2009, 23(5):60 63

[10] LIANG Kun. Research on several crucial problems in high sensitivity 
GPS receiving techniques[D]. Beijing:Graduate University of the Chinese Academy of Sciences,2007
[11] JAMES B Y T. Fundamentals of Global Positioning System Receivers[M]. Hoboken: John Wiley \& Sons, Inc.,2005 